\title{
Modified fractional Cauchy problem in a complex domain
}

Rabha W Ibrahim*

\section{"Correspondence:}

rabhaibrahim@yahoo.com

Institute of Mathematical Sciences, University Malaya, Kuala Lumpur,

50603, Malaysia

\begin{abstract}
In this paper, we make an extension to the Srivastava-Owa fractional operators in the space $L^{2}(U ; H)$, where $U$ is the open unit disk and $H$ is a complex Hilbert space. Some recurrent relations are imposed on these extended operators. Moreover, by employing the theory of sums of accretive operators, the existence and uniqueness of the solution of the fractional Cauchy problem (in the sense of extended Srivastava-Owa fractional operators) is studied in a complex Hilbert space. Applications are illustrated.
\end{abstract}

Keywords: fractional calculus; fractional differential equations; Srivastava-Owa fractional operators; unit disk; analytic function; Cauchy problem

\section{Introduction}

Fractional derivatives can express the properties of memory and heredity of materials, which is the chief benefit of fractional derivatives compared with integer-order derivatives. Practical problems require definitions of fractional derivatives allowing the use of physically interpretable initial conditions. Fractional time derivatives are linked with irregular sub-diffusion, where a darken of particles spreads more slowly than a classical diffusion. While the fractional space derivatives are used to model irregular diffusion or dispersion, where a particle follow spreads at a rate not in agreement with the classical Brownian motion model, and the follow can be asymmetric [1-3].

Fractional differential and integro-differential equations occur from different real processes, and phenomena arise in physics such as signal processing and image processing, optics, engineering, control system, computer science (such as real neural network, complex neural network, information technology), statistics and probability, astronomy, geophysics, hydrology, chemical technology, materials, robots, earthquake analysis, electric fractal network, statistical mechanics, biotechnology, medicine, and economics [4-10].

Fractional Cauchy problems restore the integer time derivative by its fractional complement. Nigmatullin [11] posed a physical derivation of the fractional Cauchy problem; Kochubei [12] introduced the mathematical study of fractional Cauchy problems; Meerschaert et al. [13] constructed the stochastic solutions for fractional Cauchy problems in a bounded domain; Zaslavsky [14], falsified fractional Cauchy problems as a model for Hamiltonian chaos; Kexue and Jigen [15], concerned with fractional abstract Cauchy problems with order $\alpha \in(1,2)$, proposed the sufficient conditions for the existence and uniqueness of mild solutions and strong solutions of the inhomogeneous fractional Cauchy problem; Li et al. [16] established an existence theorem for mild solutions to the nonlocal

(c) 2013 Ibrahim; licensee Springer. This is an Open Access article distributed under the terms of the Creative Commons Attribution License (http://creativecommons.org/licenses/by/2.0), which permits unrestricted use, distribution, and reproduction in any medium, provided the original work is properly cited. 
Cauchy problem by virtue of measure of noncompactness and the fixed point theorem for condensing maps; Zhong et al. [17] studied the Cauchy problem for some local fractional abstract differential equation with fractal conditions; Yang [18] considered the problem for local fractional derivatives from local fractional functional analysis theory; finally, local fractional Cauchy formula within fractal complex domain was investigated in [19-21]. Recently, the author studied the fractional Cauchy problem in a complex domain [22-26].

In this article, we shall make an extension to the Srivastava-Owa fractional operators in the space $L^{2}(U ; H)$, where $U$ is the open unit disk and $H$ is a complex Hilbert space. Some properties are discussed such as the recurrent relations. Moreover, by applying the theory of sums of accretive operators, the existence and uniqueness of the solution of the fractional Cauchy problem (in the sense of extended Srivastava-Owa fractional operators) is established in a complex Hilbert space. Applications are introduced.

\section{Fractional calculus}

In [27], Srivastava and Owa provided the definitions for fractional operators (derivative and integral) in the complex $z$-plane $\mathbb{C}$ as follows.

Definition 2.1 The fractional derivative of order $\alpha$ is defined for a function $f(z)$ by

$$
D_{z}^{\alpha} f(z):=\frac{1}{\Gamma(1-\alpha)} \frac{d}{d z} \int_{0}^{z} \frac{f(\zeta)}{(z-\zeta)^{\alpha}} d \zeta, \quad 0 \leq \alpha<1
$$

where the function $f(z)$ is analytic in a simply-connected region of the complex $z$-plane $\mathbb{C}$ containing the origin, and the multiplicity of $(z-\zeta)^{-\alpha}$ is removed by requiring $\log (z-\zeta)$ to be real when $(z-\zeta)>0$.

Definition 2.2 The fractional integral of order $\alpha$ is defined, for a function $f(z)$, by

$$
I_{z}^{\alpha} f(z):=\frac{1}{\Gamma(\alpha)} \int_{0}^{z} f(\zeta)(z-\zeta)^{\alpha-1} d \zeta, \quad \alpha>0
$$

where the function $f(z)$ is analytic in a simply-connected region of the complex $z$-plane $(\mathbb{C})$ containing the origin, and the multiplicity of $(z-\zeta)^{\alpha-1}$ is removed by requiring $\log (z-\zeta)$ to be real when $(z-\zeta)>0$.

Remark 2.1 From Definitions 2.1 and 2.2, we have

$$
D_{z}^{\alpha} z^{\beta}=\frac{\Gamma(\beta+1)}{\Gamma(\beta-\alpha+1)} z^{\beta-\alpha}, \quad \beta>-1,0 \leq \alpha<1
$$

and

$$
I_{z}^{\alpha} z^{\beta}=\frac{\Gamma(\beta+1)}{\Gamma(\beta+\alpha+1)} z^{\beta+\alpha}, \quad \beta>-1, \alpha>0 .
$$

In this note, we are concerned about the following fractional Cauchy problem (in the sense of the Srivastava-Owa operator):

$$
\begin{aligned}
& D_{z}^{\alpha} u(z)+C(z) A u(z)=\phi(z), \quad \alpha \in(0,1), \\
& \left.I_{z}^{1-\alpha} u(z)\right|_{z=0}=0,
\end{aligned}
$$


where $A: D(A)=: \widetilde{H} \rightarrow H$ is a closed densely defined linear operator on a complex Hilbert space $H, C(\cdot)$ is a bounded operator defined everywhere in $L^{2}(U ; H), \phi \in L^{2}(U ; H)$ and $u \in$ $L^{2}(U ; \widetilde{H}), \widetilde{H} \subset H, U:=\{z \in \mathbb{C}:|z|<1\}$. Denote by $\partial U:=\{z \in \mathbb{C}:|z|=1\}$ and $\bar{U}:=U \cup \partial U$. For complex Hilbert spaces $H^{*}$ and $H$ with the inner product $(\cdot)_{H}$ and $(\cdot)_{H^{*}}$ respectively, let $B\left(H^{*}, H\right)$ be the space of all bounded linear operators from $H^{*}$ to $H$; if $H=H^{*}$, we write $B(H)$. Recall that the operator $P$ is called accretive if $\Re(P u, u)_{H} \geq 0, \forall u \in H^{*}$, and $m$ accretive if $\operatorname{Rang}(\lambda I+P)=H, \lambda>0$. Denoted by $\rho(A):=\{\lambda \in \mathbb{C}: \lambda$ is a regular value of $A\}$, the resolvent set of the operator $A$. Note that the resolvent set $\rho(A) \subseteq \mathbb{C}$ of a bounded linear operator $A$ is an open set. Moreover, the space $L^{2}(U ; H)$ is a Hilbert space with the inner product

$$
(f, g)_{L^{2}(U ; H)}=\int_{0}^{1}(f(z), g(z))_{H} d z, \quad z \in U
$$

Throughout the paper, we consider $\Re(A u, u)_{H}>0, u \in \widetilde{H}$ and $\|x\|_{\tilde{H}}=\|A x\|_{H}$.

Definition 2.3 Equation (1) has maximal regularity in $L^{2}(U ; H)$ if for every $\phi \in L^{2}(U ; H)$, $\exists u(z)$ such that

$$
u \in L^{2}(U ; \widetilde{H}), \quad I_{z}^{1-\alpha} u \in W_{0}^{1,2}(U, H)
$$

where $W_{0}^{1,2}(U, H)$ is the Sobolev space defined by

$$
W_{0}^{1,2}(U, H)=\left\{f: \exists \psi \in L^{2}(U ; H) ; f(z)=\int_{0}^{z} \psi(\zeta) d \zeta, \zeta \in U\right\}
$$

or

$$
W_{0}^{1,2}(U, H)=\left\{f: \exists \psi \in L^{2}(U ; H) ; f(z) \in L^{1}(U, \psi)\right\} .
$$

By employing the concept of sums of accretive operators, we shall prove the maximal regularity of problem (1).

We proceed to extend the fractional integral operator $I_{z}^{\alpha}$ to the space $L^{2}(U ; H)$. Define the fractional integral operator $\mathcal{I}_{z}^{\alpha}: L^{2}(U ; H) \rightarrow L^{2}(U ; H)$ by

$$
\mathcal{I}_{z}^{\alpha} u(z):=\int_{0}^{z} \frac{(z-\zeta)^{\alpha-1}}{\Gamma(\alpha)} u(\zeta) d \zeta, \quad \alpha>0
$$

where $u \in L^{2}(U ; H)$. We have the following property.

Lemma 2.1 $\mathcal{I}_{z}^{\alpha} \in B\left(L^{2}(U ; H)\right)$.

Proof By making use of the Young inequality, it follows that

$$
\left\|\mathcal{I}_{z}^{\alpha}\right\|_{L^{2}(U ; H)} \leq\left\|\mu_{\alpha}\right\|_{L^{1}(U)}\|u\|_{L^{2}(U ; H)} \leq C\|u\|_{L^{2}(U ; H)} .
$$


Similarly, we extend the fractional integral operator $D_{z}^{\alpha}$ to the space $L^{2}(U ; H)$ by the operator

$$
\mathcal{D}_{z}^{\alpha}: L^{2}(U ; H) \rightarrow L^{2}(U ; H)
$$

such that

$$
\mathcal{D}_{z}^{\alpha} u(z):=\frac{1}{\Gamma(1-\alpha)} \frac{d}{d z} \int_{0}^{z} \frac{u(\zeta)}{(z-\zeta)^{\alpha}} d \zeta, \quad 0 \leq \alpha<1 .
$$

Furthermore, we define the space $\mathcal{W}_{0}^{\alpha, 2}(U, H)$ as follows:

$$
\mathcal{W}_{0}^{\alpha, 2}(U, H):=\left\{u \in L^{2}(U ; H): I_{z}^{1-\alpha} u \in W_{0}^{1,2}(U, H)\right\}
$$

Lemma 2.2 Let $f \in L^{2}(U ; H)$, then

$$
\mathcal{I}_{z}^{\alpha+\beta} f=\mathcal{I}_{z}^{\alpha} \mathcal{I}_{z}^{\beta} f, \quad \alpha>0, \beta>0
$$

Proof For a function $f$, using the Dirichlet technique yields

$$
\begin{aligned}
\mathcal{I}_{z}^{\alpha} \mathcal{I}_{z}^{\beta} f(z) & =\frac{1}{\Gamma(\alpha)} \int_{0}^{z}(z-\zeta)^{\alpha-1} \mathcal{I}_{\zeta}^{\beta} f(\zeta) d \zeta \\
& =\frac{1}{\Gamma(\alpha) \Gamma(\beta)} \int_{0}^{z}(z-\zeta)^{\alpha-1}\left(\int_{\xi}^{\zeta}(\zeta-\xi)^{\beta-1} f(\xi)\right) d \xi d \zeta \\
& =\frac{1}{\Gamma(\alpha) \Gamma(\beta)} \int_{0}^{z} f(\xi)\left(\int_{\xi}^{\zeta}(z-\zeta)^{\alpha-1}(\zeta-\xi)^{\beta-1}\right) d \zeta d \xi
\end{aligned}
$$

Let $\omega:=\frac{\zeta-\xi}{z-\xi}$, we impose

$$
\begin{aligned}
\int_{\xi}^{\zeta}(z-\zeta)^{\alpha-1}(\zeta-\xi)^{\beta-1} d \zeta & =(z-\xi)^{\alpha+\beta-1} \int_{0}^{1}(1-\omega)^{\alpha-1} \omega^{\beta-1} d \omega \\
& =(z-\xi)^{\alpha+\beta-1} \frac{\Gamma(\alpha) \Gamma(\beta)}{\Gamma(\alpha+\beta)}
\end{aligned}
$$

Thus we have

$$
\begin{aligned}
\mathcal{I}_{z}^{\alpha} \mathcal{I}_{z}^{\beta} f(z) & =\frac{1}{\Gamma(\alpha) \Gamma(\beta)} \int_{0}^{z}(z-\zeta)^{\alpha+\beta-1} \frac{\Gamma(\alpha) \Gamma(\beta)}{\Gamma(\alpha+\beta)} f(\xi) d \xi \\
& =\frac{1}{\Gamma(\alpha) \Gamma(\beta)} \int_{0}^{z}(z-\xi)^{\alpha+\beta-1} \frac{\Gamma(\alpha) \Gamma(\beta)}{\Gamma(\alpha+\beta)} f(\xi) d \xi \\
& =\frac{1}{\Gamma(\alpha+\beta)} \int_{0}^{z}(z-\xi)^{\alpha+\beta-1} f(\xi) d \xi \\
& =\mathcal{I}_{z}^{\alpha+\beta} f(z) .
\end{aligned}
$$

Lemma 2.3 $\mathcal{D}_{z}^{\alpha} \mathcal{I}_{z}^{\alpha} u(z)=u(z)$. 
Proof By Lemma 2.2, we have

$$
\mathcal{D}_{z}^{\alpha} \mathcal{I}_{z}^{\alpha} u(z)=\mathcal{D}_{z}^{1} \mathcal{I}_{z}^{1-\alpha} \mathcal{I}_{z}^{\alpha} u(z)=\mathcal{D}_{z}^{1} \mathcal{I}_{z}^{1} u(z)=u(z)
$$

From the last assertion, we conclude that $\mathcal{D}_{z}^{1}=\mathcal{I}_{z}^{-1}$.

Lemma 2.4 Let $\alpha \in[k-1, k)$, then $\mathcal{I}_{z}^{\alpha} \mathcal{D}_{z}^{\alpha} u(z)=u(z)-\sum_{j=1}^{k}\left[\mathcal{D}_{z}^{\alpha-j} u(z)\right]_{z=0} \frac{z^{\alpha-j}}{\Gamma(\alpha-j+1)}$.

Proof Since

$$
\begin{aligned}
\mathcal{I}_{z}^{\alpha} \mathcal{D}_{z}^{\alpha} u(z) & =\frac{1}{\Gamma(\alpha)} \int_{0}^{z}(z-\zeta)^{\alpha-1} \mathcal{D}_{z}^{\alpha} u(\zeta) d \zeta \\
& =\frac{d}{d z}\left[\frac{1}{\Gamma(\alpha+1)} \int_{0}^{z}(z-\zeta)^{\alpha} \mathcal{D}_{z}^{\alpha} u(\zeta) d \zeta\right]
\end{aligned}
$$

then, by using integration by parts, we get

$$
\begin{aligned}
\frac{1}{\Gamma(\alpha+1)} \int_{0}^{z}(z-\zeta)^{\alpha} \mathcal{D}_{z}^{\alpha} u(\zeta) d \zeta= & \frac{1}{\Gamma(\alpha+1)} \int_{0}^{z}(z-\zeta)^{\alpha} \frac{d^{k}}{d \zeta^{k}} \mathcal{D}_{z}^{-(k-\alpha)} u(\zeta) d \zeta \\
= & \frac{1}{\Gamma(\alpha-k+1)} \int_{0}^{z}(z-\zeta)^{\alpha-k} \mathcal{D}_{z}^{-(k-\alpha)} u(\zeta) d \zeta \\
& -\sum_{j=1}^{k} \frac{d^{k-j}}{d \zeta^{k-j}}\left[\mathcal{D}_{z}^{-(k-\alpha)} u(z)\right]_{z=0} \frac{z^{\alpha-j+1}}{\Gamma(\alpha-j+2)} \\
= & \frac{1}{\Gamma(\alpha-k+1)} \int_{0}^{z}(z-\zeta)^{\alpha-k} \mathcal{D}_{z}^{-(k-\alpha)} u(\zeta) d \zeta \\
& -\sum_{j=1}^{k}\left[\mathcal{D}_{z}^{\alpha-j} u(z)\right]_{z=0} \frac{z^{\alpha-j+1}}{\Gamma(\alpha-j+2)} \\
= & \mathcal{I}_{z}^{\alpha-k+1}\left(\mathcal{D}_{z}^{-(k-\alpha)} u(z)\right)-\sum_{j=1}^{k}\left[\mathcal{D}_{z}^{\alpha-j} u(z)\right]_{z=0} \frac{z^{\alpha-j+1}}{\Gamma(\alpha-j+2)} \\
= & \mathcal{I}_{z}^{1} u(z)-\sum_{j=1}^{k}\left[\mathcal{D}_{z}^{\alpha-j} u(z)\right]_{z=0} \frac{z^{\alpha-j+1}}{\Gamma(\alpha-j+2)}
\end{aligned}
$$

Combining the last two assertions, we end the proof.

Remark 2.2 For a special case $\alpha \in(0,1)$, we have the relation

$$
\mathcal{I}_{z}^{\alpha} \mathcal{D}_{z}^{\alpha} u(z)=u(z)-\left[\mathcal{D}_{z}^{\alpha-1} u(z)\right]_{z=0} \frac{z^{\alpha-1}}{\Gamma(\alpha)}
$$

Note that the initial condition of problem (1) implies that $u(z)$ of the form

$$
u(z)=\sum_{n=1}^{\infty} a_{n} z^{n}, \quad z \in U
$$


(this class of analytic functions has wide applications in the geometric function theory and the univalent function theory when $a_{1}=1$ (see [28])); hence we obtain

$$
\mathcal{I}_{z}^{\alpha} \mathcal{D}_{z}^{\alpha} u(z)=u(z), \quad \alpha \in(0,1)
$$

By virtue of the last discussion, we have the following result.

Lemma 2.5 Let $u \in L^{2}(U ; H)$, then $\mathcal{D}_{z}^{\alpha} u(z), \alpha \in(0,1)$ is an accretive operator.

Proof To prove that $\mathcal{D}_{z}^{\alpha} u(z)$ is an accretive operator, it suffices to show that $\Re\left(\mathcal{D}_{z}^{\alpha} u\right.$, $u)_{L^{2}(U ; H)} \geq 0$, where $u$ is in the domain of $\mathcal{D}_{z}^{\alpha}$. By the definition of $\mathcal{I}_{z}^{\alpha} u(z)$, we receive that $\Re\left((z-\zeta)^{\alpha-1}\right)>0$; consequently, this implies that

$$
\Re\left(\mathcal{I}_{z}^{\alpha} v, v\right)_{L^{2}(U ; H)} \geq 0
$$

where $v \in L^{2}(U ; H)$. Hence, by Remark 2.2, we have

$$
\Re\left(\mathcal{D}_{z}^{\alpha} u, u\right)_{L^{2}(U ; H)}=\Re\left(\mathcal{D}_{z}^{\alpha} u, \mathcal{I}_{z}^{\alpha} \mathcal{D}_{z}^{\alpha} u\right)_{L^{2}(U ; H)}:=\Re\left(u^{(\alpha)}, \mathcal{I}_{z}^{\alpha} u^{(\alpha)}\right)_{L^{2}(U ; H)} \geq 0
$$

but $u$ is in the domain of $\mathcal{D}_{z}^{\alpha}$, so, consequently, $\mathcal{D}_{z}^{\alpha} u(z)$ is an accretive operator.

Lemma 2.6 Let $u \in L^{2}(U ; H)$, then $\mathcal{D}_{z}^{\alpha} u(z)$ is an m-accretive operator.

Proof To prove that $\operatorname{Rang}\left(\lambda I+\mathcal{D}_{z}^{\alpha}\right)=H$, it suffices to show that the function $\left(\lambda I+\mathcal{D}_{z}^{\alpha}\right)^{-1} \phi$ is well defined for all $\lambda>0, u \in L^{2}(U ; H)$ and bounded in $L^{2}(U ; H)$. A simple computation shows that

$$
\Phi(z):=\left[\left(\lambda I+\mathcal{D}_{z}^{\alpha}\right)^{-1} \phi\right](z)=\int_{0}^{z}(z-\zeta)^{\alpha-1} E_{\alpha, \alpha}\left(-\lambda(z-\zeta)^{\alpha}\right) \phi(\zeta) d \zeta,
$$

where $\phi \in L^{2}(U ; H)$ and

$$
E_{\alpha, \beta}:=\sum_{m=0}^{\infty} \frac{z^{m}}{\Gamma(m \alpha+\beta)}
$$

is a Mittag-Leffler function. Therefore, by applying the Young inequality, we conclude that

$$
\|\Phi(z)\|_{L^{2}(U ; H)} \leq L_{\alpha, \lambda}\|\phi\|_{L^{2}(U ; H)}, \quad|z|<1,
$$

where $L_{\alpha, \lambda}>0$. Thus $\Phi(z)$ is well defined for all $\lambda>0, u \in L^{2}(U ; H)$ and bounded in $L^{2}(U ; H)$. This implies that $\mathcal{D}_{z}^{\alpha} u(z)$ is an $m$-accretive operator.

\section{Existence and uniqueness}

In this section, we study the maximal regularity of the fractional Cauchy problem (1)

$$
\begin{aligned}
& D_{z}^{\alpha} u(z)+C(z) A u(z)=\phi(z), \quad \alpha \in(0,1), \\
& \left.I_{z}^{1-\alpha} u\right|_{z=0}=0,
\end{aligned}
$$

under the following assumptions. 
(H1) $A$ is a linear $m$-accretive operator in $H, 0 \in \rho(A)$, where $H$ is a complex Hilbert space.

(H2) $C$ is a bounded operator in $L^{2}(U ; H)$ and there exists $\mu>0$ with

$$
\Re(C(\cdot) u, u)_{L^{2}(U ; H)} \geq \mu\|u\|_{L^{2}(U ; H)}^{2}
$$

and $u \in \widetilde{H}, \widetilde{H} \subset H$.

(H3) $\phi \in L^{2}(U ; H)$.

We provide the main result of this section. We need the following result.

Lemma 3.1 [29] Let $P$ be $m$-accretive and let $Q$ be accretive Lipschitz continuous in a Hilbert space $H$. Then $P+Q$ is m-accretive with $\operatorname{dom}(P+Q)=\operatorname{dom}(P)$.

Theorem 3.1 Let the assumptions (H1)-(H3) hold. Then there exists a unique function $u \in \mathcal{W}_{0}^{\alpha, 2}(U, H) \cap L^{2}(U ; \widetilde{H})$ satisfying $(1)$ and the inequality

$$
\|u\|_{L^{2}(U ; H)}+\left\|D_{z}^{\alpha} u\right\|_{L^{2}(U ; H)}+\|A u\|_{L^{2}(U ; H)} \leq K\|\phi\|_{L^{2}(U ; H)}, \quad K>0 .
$$

Proof Our aim is to rewrite Eq. (1) as an operator equation in $L^{2}(U ; H)$. For this purpose, we define the following operators in $L^{2}(U ; H)$ :

$$
(\mathfrak{A} u)(z):=A u(z), \quad u \in \operatorname{dom}(\mathfrak{A})=L^{2}(U ; \widetilde{H})
$$

and

$$
(\mathfrak{C} u)(z):=C(z) u(z), \quad \operatorname{dom}(\mathfrak{C})=L^{2}(U ; H)
$$

thus Eq. (1) becomes

$$
\mathcal{D}_{z}^{\alpha} u+\mathfrak{C} \mathfrak{A} u=\phi
$$

Since $0 \in \rho(A)(\mathrm{H} 1)$, therefore, we let $u=\mathfrak{A}^{-1} w$, then we pose

$$
\mathcal{D}_{z}^{\alpha} \mathfrak{A}^{-1} w+\mathfrak{C} w=\phi
$$

Our point is to prove that (9) has a unique solution. It suffices to show that $\mathcal{D}_{z}^{\alpha} \mathfrak{A}^{-1}$ is $m$ accretive. For the accretivity, we must show that

$$
\mathfrak{R}\left(\mathcal{D}_{z}^{\alpha} \mathfrak{A}^{-1} w, w\right)_{L^{2}(U ; H)} \geq 0, \quad w \in \operatorname{dom}\left(\mathcal{D}_{z}^{\alpha} \mathfrak{A}^{-1}\right),
$$

where

$$
\operatorname{dom}\left(\mathcal{D}_{z}^{\alpha} \mathfrak{A}^{-1}\right):=\left\{u \in L^{2}(U ; H): \mathfrak{A}^{-1} u \in \operatorname{dom}\left(\mathcal{D}_{z}^{\alpha}\right)\right\}
$$

According to Lemma 2.5 and Lemma 2.6, we obtain that $\mathcal{D}_{z}^{\alpha} \mathfrak{A}^{-1}$ is $m$-accretive. Moreover, by $(\mathrm{H} 2)$ it follows that the operator $\mathfrak{C}-\mu$ is a bounded accretive operator. By virtue of 
Lemma 3.1, it follows that $\mathcal{D}_{z}^{\alpha} \mathfrak{A}^{-1}+\mathfrak{C}-\mu, \mu>0$, is $m$-accretive. Together with (H3), we obtain that Eq. (9) has a unique solution $w \in L^{2}(U ; H)$.

Next, we proceed to prove the inequality (7). By taking the inner product of (9) with $w$ and using the accretivity of the operators $\mathcal{D}_{z}^{\alpha} \mathfrak{A}^{-1}$ and $\mathfrak{C}-\mu$, we observe that

$$
\Re(\phi, w)_{L^{2}(U ; H)} \geq \mu\|w\|_{L^{2}(U ; H)}
$$

and by applying the Cauchy-Schwarz inequality, we obtain

$$
\|w\|_{L^{2}(U ; H)} \leq \frac{1}{\mu}\|\phi\|_{L^{2}(U ; H)} .
$$

Moreover, by the boundedness of the operator $\mathfrak{C}$, there exists a constant $\Lambda>0$ such that

$$
\left\|\mathcal{D}_{z}^{\alpha} \mathfrak{A}^{-1} w\right\|_{L^{2}(U ; H)}+\|w\|_{L^{2}(U ; H)} \leq \Lambda\|\phi\|_{L^{2}(U ; H)} .
$$

Consequently, we have

$$
\left\|\mathcal{D}_{z}^{\alpha} u\right\|_{L^{2}(U ; H)}+\|\mathfrak{A} u\|_{L^{2}(U ; H)} \leq \Lambda\|\phi\|_{L^{2}(U ; H)} .
$$

Since $\mathcal{I}_{z}^{\alpha}$ is bounded in $L^{2}(U ; H)$ (Lemma 2.1) and by the fact that $u=\mathcal{I}_{z}^{\alpha} \mathcal{D}_{z}^{\alpha} u$, it follows

$$
\|u\|_{L^{2}(U ; H)}=\left\|\mathcal{I}_{z}^{\alpha} \mathcal{D}_{z}^{\alpha} u\right\|_{L^{2}(U ; H)} \leq \Lambda_{1}\left\|\mathcal{D}_{z}^{\alpha} u\right\|_{L^{2}(U ; H)} .
$$

Hence, from the last two inequalities, we conclude the inequality (9). This completes the proof.

Corollary 3.1 Let $A$ be accretive and self-adjoint in $H, 0 \in \rho(A)$ and let the hypotheses $(\mathrm{H} 2)$ and $(\mathrm{H} 3)$ hold. Then there exists a unique function $u \in \mathcal{W}_{0}^{\alpha, 2}(U, H) \cap L^{2}(U ; \widetilde{H})$ satisfying (1) and the inequality (7).

Proof It is well known that an accretive and self-adjoint operator implies a symmetric and $m$-accretive one [30]. Hence, by virtue of Theorem 3.1, there exists a unique function $u \in \mathcal{W}_{0}^{\alpha, 2}(U, H) \cap L^{2}(U ; \widetilde{H})$ satisfying (1) and the inequality (7).

Corollary 3.2 Let C be accretive and Lipschitz continuous in $H$ and let the hypotheses (H1) and $(\mathrm{H} 3)$ hold. Then there exists a unique function $u \in \mathcal{W}_{0}^{\alpha, 2}(U, H) \cap L^{2}(U ; \widetilde{H})$ satisfying $(1)$ and the inequality (7).

Proof Since $C$ is Lipschitz continuous in $H$, then for all $u_{1}$ and $u_{2} \in L^{2}(U ; H)$ we have

$$
\left\|C(z) u_{1}-C(z) u_{2}\right\|_{L^{2}(U ; H)} \leq \gamma_{1}\left\|u_{1}-u_{2}\right\|_{L^{2}(U ; H)} .
$$

Consequently, we get

$$
\Re\left(C(z) u_{1}-C(z) u_{2}, u_{1}-u_{2}\right) \geq \gamma_{2}\left\|u_{1}-u_{2}\right\|_{L^{2}(U ; H)}^{2},
$$


where $\gamma_{1}$ and $\gamma_{2}$ are positive; thus in view of Theorem 3.1, there exists a unique function $u \in \mathcal{W}_{0}^{\alpha, 2}(U, H) \cap L^{2}(U ; \widetilde{H})$ satisfying (1) and the inequality (7).

Example 3.1 Consider the problem

$$
\begin{aligned}
& D_{z}^{\alpha} u(z)+\lambda\left(h\left(u_{z}\right)\right)_{z}=\phi(z), \quad \alpha \in(0,1), \\
& \left.I_{z}^{1-\alpha} u\right|_{z=0}=0
\end{aligned}
$$

where $u(0)=u(1)=0, u \in L^{2}(U ; H), 0<\Re h<\infty, 0<\Re h^{\prime}<\infty, \lambda \in \mathbb{C}$ and $\phi \in L^{2}(U ; H)$. Obviously, by employing a fixed point argument to the linearized equation, we receive

$$
\lambda\left(h\left(u_{z}\right)\right)_{z}=\lambda h^{\prime}\left(u_{z}\right) u_{z z}:=\lambda h^{\prime}(w) u_{z z}
$$

thus we can define

$$
A u:=\lambda u_{z z}, \quad C(z) u:=h^{\prime}(w) u
$$

such that

$$
\operatorname{dom}(A)=\left\{g \in W^{2,2}(U): g(0)=g(1)=0\right\}
$$

where

$$
W^{2,2}(U)=\left\{f \in L^{2}(U), f^{\prime \prime} \in L^{2}(U)\right\}
$$

It follows that $A$ and $C$ satisfy the conditions of Corollary 3.1 for some $\lambda$, and therefore (10) has a unique function $u \in \mathcal{W}_{0}^{\alpha, 2}(U, H) \cap L^{2}(U ; \tilde{H})$.

Note that self-adjoint operators on a Hilbert space are applied in quantum mechanics to describe a physical observation such as the position, momentum, angular momentum and spin. The differential operators corresponding to the Legendre differential equation and the harmonic motion equation are self-adjoint, while those corresponding to the Laguerre differential equation and Hermite differential equation are not. A nonself-adjoint secondorder linear differential operator can be viewed as a self-adjoint by using Sturm-Liouville theory.

Example 3.2 Consider the problem

$$
D_{z}^{\alpha} u(z)+i u_{z}=\phi(z), \quad \alpha \in(0,1)
$$

such that $u(0)=u(1)=0$ and that $\left.I_{z}^{1-\alpha} u\right|_{z=0}=0$. Let $u, \phi \in L^{2}(U ; H)$ and

$$
A u:=i \frac{d}{d z} u, \quad C(z) u=1
$$

It is clear that $A$ is a self-adjoint operator and $C$ is bounded; thus it follows that the problem (11) has a unique solution. 


\section{Competing interests}

The author declares that she has no competing interests.

\section{Author's contributions}

The author read and approved the final manuscript.

\section{Acknowledgements}

The author is thankful to the referees for helpful suggestions for the improvement of this article. This research has been funded by the university of Malaya, under Grant No. RG208-11AFR.

\section{Received: 8 December 2012 Accepted: 6 May 2013 Published: 23 May 2013}

\section{References}

1. Jeon, J-H, Metzler, R: Fractional Brownian motion and motion governed by the fractional Langevin equation in confined geometries. Phys. Rev. E, Stat. Nonlinear Soft Matter Phys. 81, 021103 (2010)

2. Burnecki, K, Kepten, E, Janczura, J, Bronshtein, I, Garini, Y, Weron, A: Universal algorithm for identification of fractional Brownian motion. A case of telomere subdiffusion. Biophys. J. 103, 1839-1847 (2012)

3. Bojdecki, T, Gorostiza, LG, Talarczyk, A: Oscillatory fractional Brownian motion. Acta Appl. Math. (2013). doi:10.1007/s10440-013-9798-3

4. Băleanu, D, Mustafa, OG, Agarwal, RP: Asymptotically linear solutions for some linear fractional differential equations. Abstr. Appl. Anal. 2010, 865139 (2010). doi:10.1155/2010/865139

5. Băleanu, D, Mustafa, OG, Agarwal, RP: On the solution set for a class of sequential fractional differential equations. J. Phys. A 43, 385209 (2010)

6. Bǎleanu, D, Agarwal, RP, Mustafa, OG, Coşulschi, M: Asymptotic integration of some nonlinear differential equations with fractional time derivative. J. Phys. A 44, 055203 (2011)

7. Băleanu, D, Mustafa, OG, Agarwal, RP: Asymptotic integration of $(1+\alpha)$-order fractional differential equations. Comput. Math. Appl. 62(3), 1492-1500 (2011)

8. Jalab, HA, Ibrahim, RW: Texture enhancement for medical images based on fractional differential masks. Discrete Dyn. Nat. Soc. 2013, 618536 (2013). doi:10.1155/2013/618536

9. Jalab, HA, Ibrahim, RW: On generalized fractional differentiator signals. Discrete Dyn. Nat. Soc. 2013, 795954 (2013). doi:10.1155/2013/795954

10. Ibrahim, RW, Jalab, HA: The fractional complex step method. Discrete Dyn. Nat. Soc. 2013, 515973 (2013). doi:10.1155/2013/515973

11. Nigmatullin, RR: The realization of the generalized transfer in a medium with fractal geometry. Phys. Status Solidi B $133,425-430(1986)$

12. Kochubei, AN: The Cauchy problem for evolution equations of fractional order. Differ. Uravn. (Minsk) $25,1359-1368$ (1989)

13. Meerschaert, MM, Nane, E, Vellaisamy, P: Fractional Cauchy problems in bounded domains. Ann. Probab. 37, 979-1007 (2009)

14. Zaslavsky, GM: Fractional kinetic equation for Hamiltonian chaos. Physica D 76, 110-122 (1994)

15. Li, K, Jigen, P: Fractional abstract Cauchy problems. Integral Equ. Oper. Theory 70, 333-361 (2011)

16. Li, F, Liang, J, Lu, TT, Zhu, H: A nonlocal Cauchy problem for fractional integrodifferential equations. J. Appl. Math. 2012, Article ID 901942 (2012)

17. Zhong, WP, Yang, XJ, Gao, F: A Cauchy problem for some local fractional abstract differential equation with fractal conditions. J. Appl. Funct. Anal. 8(1), 92-99 (2013)

18. Yang, XJ: Advanced Local Fractional Calculus and Its Applications. World Science Publisher, New York (2012)

19. Yang, XJ: Local fractional integral transforms. Prog. Nonlinear Sci. 4, 1-225 (2011)

20. Yang, XJ: Local Fractional Functional Analysis and Its Applications. Asian Academic Publisher, Hong Kong (2011)

21. Guo, Y: Local fractional Z transform in fractal space. Adv. Digit. Multimed. 1(2), 96-102 (2012)

22. Ibrahim, RW, Darus, M: Subordination and superordination for univalent solutions for fractional differential equations. J. Math. Anal. Appl. 345, 871-879 (2008)

23. Ibrahim, RW: Solutions of fractional diffusion problems. Electron. J. Differ. Equ. 147, 1-11 (2010)

24. Ibrahim, RW: Existence of fractional parametric Cauchy problem. Appl. Sci. 12, 30-36 (2010)

25. Ibrahim, RW: Existence and uniqueness of holomorphic solutions for fractional Cauchy problem. J. Math. Anal. Appl. 380, 232-240 (2011)

26. Ibrahim, RW: On generalized Srivastava-Owa fractional operators in the unit disk. Adv. Differ. Equ. 2011, Article ID 55 (2011)

27. Srivastava, HM, Owa, S: Univalent Functions, Fractional Calculus, and Their Applications. Wiley, New York (1989)

28. Duren, PL: Univalent Functions. Springer, Berlin (1983)

29. Brezis, H: Opérateurs Maximaux Monotones et Semi-groupes de Contractions dans les Espaces de Hilbert. Math. Studies, vol. 5. North-Holland, Amsterdam (1973)

30. Bajlekova, E: Fractional evolution equations in Banach spaces. PhD thesis, Eindhoven University of Technology. http://alexandria.tue.nl/extra2/200113270.pdf (2001) 